NASA/TM-1999-209280

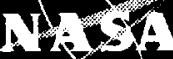

A Precise Calibration Technique for Measuring High Gas Temperatures

Suleyman A. Gokoglu and Donald F. Schultz

Glenn Research Center, Cleveland, Ohio

Prepared for the

Mediterranean Combustion Symposium

cosponsored by the Combustion Institute and

International Centre for Heat and Mass Transfer

Antalya, Turkey, June 20-25, 1999

National Aeronautics and

Space Administration

Glenn Research Center 


\section{Acknowledgments}

We thank Dr. A. Sayir for providing the fibers and for sharing his experience on fibers during their growth. We appreciate the assistance of Dr. S. Farmer for taking the photographs of the fiber molten tips.

We thank D. Hunter and Prof. C. Adler for their help with the IR data.

Available from

NASA Center for Aerospace Information

7121 Standard Drive

Hanover, MD 21076

Price Code: A03
National Technical Information Service 5285 Port Royal Road Springfield, VA 22100 Price Code: A03 


\title{
1A PRECISE CALIBRATION TECHNIQUE FOR MEASURING HIGH GAS TEMPERATURES
}

\author{
Suleyman A. Gokoglu and Donald F. Schultz \\ NASA Glenn Research Center \\ Cleveland, Ohio 44135
}

\begin{abstract}
A technique was developed for direct measurement of gas temperatures in the range of $2050 \mathrm{~K}-$ $2700 \mathrm{~K}$ with improved accuracy and reproducibility. The technique utilized the low-emittance of certain fibrous materials, and the uncertainty of the technique was limited by the uncertainty in the melting points of the materials, i.e., $\pm 15 \mathrm{~K}$. The materials were pure, thin, metal-oxide fibers whose diameters varied from $60 \mu \mathrm{m}$ to $400 \mu \mathrm{m}$ in the experiments. The sharp increase in the emittance of the fibers upon melting was utilized as indication of reaching a known gas temperature. The accuracy of the technique was confirmed by both calculated low emittance values of transparent fibers, of order 0.01 , up to a few degrees below their melting point and by the fiber-diameter independence of the results. This melting-point temperature was approached by increments not larger than $4 \mathrm{~K}$, which was accomplished by controlled increases of reactant flow rates in hydrogen-air and/or hydrogen-oxygen flames. As examples of the applications of the technique, the gas-temperature measurements were used (a) for assessing the uncertainty in inferring gas temperatures from thermocouple measurements, and (b) for calibrating an IR camera to measure gas temperatures. The technique offers an excellent calibration reference for other gas-temperature measurement methods to improve their accuracy and reliably extending their temperature range of applicability.
\end{abstract}

\section{INTRODUCTION}

Accurate measurement of gas temperatures typically above $1500 \mathrm{~K}$ (referred to as "high" temperatures hereafter) is challenging. Thermocouple measurements at such high temperatures are not only perturbing, but also require corrections to account for the difference between the actual gas temperature and the measured thermocouple material temperature. These differences are typically larger than $100 \mathrm{~K}$ (around $300 \mathrm{~K}$ for $2000 \mathrm{~K}$ [1]) due mainly to radiative heat losses of the thermocouple. Furthermore, the uncertainties involved in determining the optical and thermo-physical properties of the gas and the thermocouple material; and in the heat transfer calculations reduce the reliability of these corrections. Moreover, the physical and chemical stability of thermocouples to withstand the thermal loads and reactive environments prevailing at high temperatures naturally limit their life and maximum temperature of use. Aspirated thermocouples, though typically more durable and shielded for reducing radiative heat loss, make gas-temperature measurements even more intrusive because they require a lower pressure sink to enable gas aspiration over the thermocouple junction. Hence, the utility of thermocouples for inferring high gas temperatures to a level of accuracy better than $\pm 50 \mathrm{~K}$ is quite limited. 
The use of optical techniques for measuring high gas temperatures has different drawbacks for accuracy. Methods based on emission pyrometry by utilizing thin fibers, though minimally perturbing due to their small size, still require well-characterized emissivity information and elimination of spectral interference. Implicit optical systems based on gas density and refractive index measurements require known gas compositions, which are dependent on temperature and chemical reactions and harder to determine at higher temperatures. Other optical techniques rely on accurate spectral information for the emissivity/absorptivity of the gas mixture constituents and theoretical temperature-dependent population balance calculations to determine temperature. They also generally require calibrations, which involve many times the use of thermocouples, and hence, the resulting measurements suffer from the same accuracy and peak temperature limitations mentioned above. For recent combustion research, even a sophisticated method involving the use of coherent anti-Stokes Raman scattering (CARS) resulted in a temperature accuracy of no better than $2.5 \%$ on a flat flame burner, i.e., larger than $\pm 50 \mathrm{~K}$ for $2000 \mathrm{~K}$ [2].

This work exploits the low emittances of optically thin materials (of the order of 0.01 until they melt) for a direct determination of gas temperatures at their melting points more accurately than other previously known techniques. The current technique has been demonstrated under atmospheric pressure by using four different oxide materials: alumina $\left(\mathrm{Al}_{2} \mathrm{O}_{3}\right.$, melting point: $2320 \mathrm{~K}$ ), ytrria $\left(\mathrm{Y}_{2} \mathrm{O}_{3}\right.$, melting point: $\left.2690 \mathrm{~K}\right)$, yttria alumina garnet (YAG, melting point: $2200 \mathrm{~K}$ ), and alumina/YAG eutectic (melting point: 2095K) [3 - 5]. For the gas temperature range covered in this study $(\sim 2050 \mathrm{~K}-2700 \mathrm{~K})$, our measurement uncertainty was only limited by the uncertainties reported in the literature for the melting points of these materials; i.e., typically $\pm 15 \mathrm{~K}[3-5]$. These four materials have been grown as pure (at least 99.999\%), transparent, thin fibers with a crystalline structure by using the laser-heated floating zone method [6]. The measured melting-point uncertainties during their growth were consistent with the literature values, except for yttria where the uncertainty was as large as $\pm 30 \mathrm{~K}$ relative to the $\pm 11 \mathrm{~K}$ reported in [5]. Because the fibers are high-temperature oxide materials, they are highly immune to chemical reactions and sublimation in conventional combustion environments and preserve their structural integrity. The diameter of the fibers used in this study varied between $60 \mu \mathrm{m}$ to $160 \mu \mathrm{m}$, but the results were found to be independent of fiber diameter, confirming that fiber emittances were sufficiently small to ascertain the accuracy of our technique. The fibers were stiff and strong enough to be placed horizontally in cross-stream of up-flowing hot gases (Reynolds number $<50$ ) and were able to withstand any shearing and gravitational forces until their melting point. However, as soon as they changed phase and melted, their optical properties changed drastically such that they became highly emissive and much brighter [7-8]. The visual observation of their melting, and the associated abrupt change in their emittance, was used as the indicator of the gas temperature in the immediate vicinity of the fiber.

The technique currently lends itself for gas-temperature measurement only under controlled and relatively benign environments and at a relatively few discrete conditions as described below. Hence, it can most effectively be utilized to calibrate other gas-temperature measurement techniques, much improving their accuracy especially in higher temperatures where their reliability becomes increasingly questionable. We applied it in this work to assess the accuracy of thermocouple measurements and to calibrate an IR camera with a spectral range from $0.4 \mu \mathrm{m}$ to $2.2 \mu \mathrm{m}$. There is no inherent difficulty for applying the technique to systems at pressures other than the atmospheric. The extension of the technique to a larger temperature range can easily be accomplished by identifying the appropriate fiber materials. 


\section{EXPERIMENTAL}

A schematic of the top-view of the experimental setup is shown in Fig. 1. A circular, vertically oriented, cooled, flat-flame burner with a flow-area radius of $1.8 \mathrm{~cm}$ was used for this study. The burner allowed stable burning of premixed hydrogen/air or hydrogen/oxygen gas mixtures under atmospheric pressure up to temperatures beyond the melting point of yttria fibers; i.e., $>2690 \mathrm{~K}$. Technical-grade hydrogen and air, or hydrogen and oxygen gas flow rates were individually adjusted to, and controlled at, desired levels prior to mixing and entering the burner. A spark igniter was placed close to the peripheral edge of the burner-top and outside the view of the IR and CCD cameras. Thin and short $(\sim 1 \mathrm{~mm}$ OD $x \sim 10 \mathrm{~cm}$ long $)$ stainless-steel tubes were used to support the fibers $(60 \mu \mathrm{m}$ to $160 \mu \mathrm{m}$ in diameter). Short portions of the fibers were inserted into the tubes from the tube ends and bonded with a high-temperature RTV adhesive. About $2 \mathrm{~cm}$ long fibers, protruding from the tube end, were inserted horizontally along the fiber axis into the flame. This length was sufficient for the bonded tip of the metallic tubes to be out of the hot zone and camera views. A remotely controlled rotational stage had four stations to hold either fiber-bearing tubes or thermocouples, as needed. Each station was capable of axial and lateral motion, allowing insertion and withdrawal of the fiber or the thermocouple to and from predetermined positions in the flame.

\section{A. Flame Temperature Profiles}

The characterization of the burner, using both thermocouple and IR band-ratio measurements, indicated that there is about a $2 \mathrm{~cm}$-long zone, between approximately $1.5 \mathrm{~cm}$ to $3.5 \mathrm{~cm}$ above the burner, where the axial temperature profile in the flow direction remains constant to within $\pm 40 \mathrm{~K}$. Hence, in this study, we fixed the height of the horizontal fiber axis above the burner at $3.0 \mathrm{~cm}$, thereby avoiding axial temperature gradients across the fiber diameter.

Typical radial temperature profiles at this height are shown in Fig. 2 for two different oxygen flow rates of $100 \mathrm{slm}$ and $120 \mathrm{slm}$ at a fixed hydrogen flow rate of $30 \mathrm{~s} / \mathrm{m}$, resulting in different flame temperatures. The measurements were made by a high-temperature-resistant $\mathrm{Ir} / \mathrm{Ir}-40 \% \mathrm{Rh}$ thermocouple with a $0.5 \mathrm{~mm}$ wire diameter. The thermocouple assembly was mounted on one of the stations of the rotational stage and programmed to traverse the flame radially where the bead started from the outside edge of the burner and returned back to the starting position. The thermocouple stopped at 6 locations, which were $0.4 \mathrm{~cm}$ apart from each other, for 40 seconds each to reach local thermal equilibrium before taking, typically, 10 readings over one second. The solid line connects the measurement points when the thermocouple is being inserted into the flame and the dashed line when it is being withdrawn from out of the flame. The standard deviation for each measurement at each location did not exceed $5 \mathrm{~K}$ for the various number of readings and frequencies tried; hence, the error bars are too small to show on the graph. The measured profiles demonstrate that the temperatures are steady and reproducible, which is indicative of a stable flame. They also show that there is a uniform temperature region at the center, which is at least $0.5 \mathrm{~cm}$ in radius, and that the temperature drops sharply towards the edge of the burner. 


\section{B. Fiber-Melting Procedure}

We inserted the tip of each fiber to slightly beyond the "knee" of the curve into the uniform temperature region; hence, most of the fiber was in the cooler region of the flame and melting occurred only at the tip of the fiber, typically not longer than $2 \mathrm{~mm}$. The fiber-tip melting was observed by CCD and IR cameras, which were isolated from the burner inside nitrogen-purged boxes. The CCD camera looked along the fiber axis with a magnifying lens and was focused on the front view of the pointing fiber tip. The IR camera looked across the fiber diameter and was focused on at least a $1 \mathrm{~cm}$-long side view of the fiber. The focusing was done for every fiber on the rotation stage before the flame was ignited. Each fiber was slowly inserted into its proper position based on the predetermined flame-temperature profile. The precise location of each station after focus was recorded to within $0.2 \times 0.2 \mathrm{~mm}^{2}$; i.e., the pixel resolution of the CCD camera. This procedure was necessary because of slightly different tube/fiber lengths and elevations during the installation. Then, the aperture of the CCD camera was set manually to the lowest level in order to protect the camera from bright images at flame conditions. Upon closing the aperture, the focused image of the transparent fiber tip disappeared in the camera. The gain and aperture settings of the IR camera, however, were always fixed, so the camera protection was provided by a remotely operated filter wheel and a shutter. The IR camera was not only used for the observation of fibers but was also calibrated as an application of this technique.

Before the flame was ignited, the fiber was completely withdrawn away from the burner area. Once a stable flame was established and its temperature was brought up close to the melting point of the specific fiber, based on our estimates from earlier thermocouple measurements and adiabatic flame temperature calculations, the fiber was reinserted slowly into its previously determined precise point of focus. This procedure protected the fiber from ignition transients and minimized its time at temperature.

We assured the stable operation of the burner by fixing the oxidant (air or oxygen) flow rate for this study at $150 \mathrm{~s} / \mathrm{m}$. This was consistent with the hydrogen/(diluted)-oxygen flame speeds reported in the literature [9-10] for the range of equivalence ratios of interest.

The precision of our measurements depended on the precision of control of the flow rates of the reactant gases while approaching the melting points of the fibers. The flow meters for both fuel and oxidant gases, which had full ranges of up to $200 \mathrm{slm}$, were controllable to within $\pm 0.1 \mathrm{~s} / \mathrm{m}$. Adiabatic flame temperature calculations using the NASA CET computer code [11] showed that the maximum flame-temperature change per $0.2 \mathrm{~s} / \mathrm{m}$ change in the hydrogen flow rate, for a fixed oxidant flow rate of $150 \mathrm{slm}$, was not larger than $4 \mathrm{~K}$. In fact, the flame temperature is less sensitive to changes in pure oxygen flow rate at a fixed hydrogen flow rate due to the combustion reaction stoichiometry, and even less sensitive to changes in air flow rate due to the additional presence of nitrogen. However, our procedure of fixing the oxidant flow rate was sufficiently precise, providing increments of no more than a maximum of $4 \mathrm{~K}$ as we approached the melting point of the fiber when the flow rate of hydrogen was increased by $0.1 \mathrm{~s} / \mathrm{m}$ each time. 
During operation, the fiber tip started to get brighter and its image reappeared on both cameras when the flame temperature got close to the fiber's melting point. The rest of the fiber was still invisible on cameras. The CCD camera image was also recorded on video for post-experiment visual analysis of fiber behavior prior to melting. The melting of the fiber tip and the formation of a tiny droplet $(\sim 1 \mathrm{~mm}$ in diameter), which was blown away by the flame, took less than one second but was easily observable on both cameras.

\section{Data Collection}

There is typically a $1 \mathrm{~mm}$-recession of the fiber length after the molten droplet is blown away. The flow rates of the reactant gases at the point of fiber-tip melting are recorded as the characteristic data pertaining to a specific fiber material. IR readings were taken within the ten seconds immediately following fiber-tip melting while the gas flow rates were carefully kept at the same constant level. For quick repetitions of the experiment, the hydrogen flow rate was reduced slightly and the fiber tip was reinserted into the same precise point of focus. Then, the hydrogen flow rate was slowly crept up again in $0.1 \mathrm{~s} / \mathrm{m}$ increments until the fiber tip melting was again observed. After the corresponding IR measurements were taken, the same sequence was repeated for multiple measurements for the same fiber. We then proceeded to continue the experiment with fibers at other stations on the rotation stage. Finally, thermocouple measurements were made at the corresponding focal positions and gas flow rates for comparisons. The identity of the fibers at each station as well as the order of the experiments, from the lowest melting-point fiber to the highest, were deliberately randomized to reassure repeatability of data.

After the molten tip of the fiber was convected away, a small drop of re-solidified material remained on the recessed fiber tip, as shown on Fig. 3, and provided physical evidence of actual melting. As can be seen from the photographs, the diameters of re-solidified drops were typically twice the diameter of the fiber, and seemed to preserve the transparent structure of the original fiber. Although the maximum diameter of the original fibers used in this study was $160 \mu \mathrm{m}$, the actual maximum diameter was sometimes as large as $400 \mu \mathrm{m}$ because we reinserted the resolidified molten tips for determining experiment repeatability and fiber-diameter dependence.

The IR camera to be calibrated was sensitive from $0.4 \mu \mathrm{m}$ up to $2.2 \mu \mathrm{m}$. The band-ratio technique described and the experimental emittance data reported in [12] were used to study the ratio of the hot $\mathrm{H}_{2} \mathrm{O}$ emission band radiance at two strong peaks centered at wavelengths of $1.45 \mu \mathrm{m}$ and $1.94 \mu \mathrm{m}$ as a function of temperature. The choice of these wavelengths was determined by the spectral responsivity of the IR camera. This also ruled out the possibility of interference from $\mathrm{CO}_{2}$ emission bands although the experiments in this study were limited to hydrogen flames. The overall uncertainty of our band ratio measurements, inclusive of all data for each fiber case, was determined to be $\pm 15 \%$.

Either air or oxygen at a fixed volumetric flow rate was used for the same hydrogen fuel in order to check the consistency of our experimental measurements. Oxygen bottles were technical grade and could be considered pure for all practical purposes; however, the oxygen content of air bottles in the experiments varied between $20 \%$ to $21 \%$ by volume. 


\section{DISCUSSION OF RESULTS}

For each oxidant gas, air or pure oxygen, at least six experiments were conducted for each fiber, except for yttria for which the material supply was limited to allow only two experiments. Alumina fibers were more readily available than the others, so data for alumina fibers represent more than fifteen experiments. The two cases for yttria fibers had to be done with pure oxygen because the maximum temperature attainable for hydrogen/air flames is not high enough to melt yttria. The experiments were randomized with respect to the oxidant gases, order of fiber material, fiber diameters or batch of fibers supplied. During the collection of data at an outdoors facility the gas-bottle temperature varied by no more than $10 \mathrm{~K}$. The standard deviations reported in the results include these random and day-to-day experimental variations.

The hydrogen flow rate at which the melting of each fiber material was observed for a fixed air or oxygen flow rate is depicted in Fig. 4. Also shown in the Figure are the results of theoretical calculations for adiabatic flame temperature as a function of hydrogen flow rate [11]. The maximum standard deviation in hydrogen flow rate for different fiber cases was determined to be $\pm 0.5 \mathrm{~s} \mathrm{~lm}$. As mentioned earlier, the maximum uncertainty in the air or oxygen flow rate, which were kept constant at $150 \mathrm{~s} / \mathrm{m}$, was $\pm 0.1 \mathrm{~s} / \mathrm{m}$. Theoretical adiabatic flame-temperature calculations for such variations in hydrogen and air, or oxygen, flow rates show that the maximum uncertainty in the corresponding flame temperatures for each fiber case is less than $\pm 15 \mathrm{~K}$. Note that the melting points of fibers were determined with a precision of better than $4 \mathrm{~K}$ in each individual experiment, as discussed above. Therefore, the overall uncertainty of our measurements is governed by the $\pm 15 \mathrm{~K}$ uncertainty in the reported melting points of these materials. Hence, the data for alumina, YAG and alumina/YAG eutectic fibers are reported in Fig. 4 as small boxes where the width of the boxes reflects the overall $\pm 0.5 \mathrm{~s} / \mathrm{m}$ standard deviation in measured hydrogen flow rates and height of the boxes reflects the $\pm 15 \mathrm{~K}$ uncertainty in the materials' melting points. For ytrria, only the averaged value for the two hydrogen flow-rate measurements is shown in Fig. 4. Also, ytrria's melting point uncertainty is plotted as $\pm 30 \mathrm{~K}$ (as opposed to $\pm 11 \mathrm{~K}$ reported in [5]) based on uncertainties determined during the growth process of this fiber using the laser-heated floating zone technique [6].

For each fiber case where both pure oxygen and air were used, the experimentally determined hydrogen flow rates at which the fiber melted for pure oxygen were consistently larger than their counterparts for air, as is shown in Fig. 4. Indeed, theoretical calculations show that this behavior is to be expected for hydrogen-lean flames; that is, pure oxygen requires more hydrogen than is necessary for air to reach the same adiabatic flame temperature when hydrogen is the deficient reactant. There are two major reasons for this difference. The first one is that the molar heat capacity of air is smaller than that of pure oxygen because of the heat-capacity difference between nitrogen and oxygen gases [13]. The second reason is that, when the volumetric (molar) flow rate of pure oxygen and air are fixed at the same level, the mass flow rate of air is smaller, due to its lighter molecular weight, than that of pure oxygen. The effect of nitrogen chemistry is found to be negligible, as expected. Naturally, the oxygen content of air determines when hydrogen is no longer the deficient reactant. The two curves for air with $20 \%$ and $21 \%$ oxygen by volume covers the range of different air bottles used in our experiments. 
Another experimental observation was that the melting points of fibers were reached when the theoretically calculated adiabatic flame temperatures were typically $100 \mathrm{~K}$ to $125 \mathrm{~K}$ higher than the respective melting points. There are two plausible explanations for this difference. The first explanation is that the flame is not adiabatic and that heat loss from the flame, especially via conduction back to the burner, may be substantial, such that the actual flame temperature may be as low as the melting point of fibers. The second explanation is that the fiber temperature may also be substantially lower than the flame temperature due to radiative cooling.

The presence of heat loss from the flame to the burner was demonstrated by the result that if the total gas flow rate was reduced, the coolant-water flow rate through the burner had to be increased to keep the burner-surface temperature-rise within safe limits. In fact, the minimum total gas flow rate was determined to be $125 \mathrm{~s} \mathrm{~m}$ for a stable operation and for avoiding flashback. Increasing the total gas flow rate for a given flame temperature should reduce this heat loss by reducing the gas-temperature gradient above the burner surface. Indeed, there seems to be a trend also in our experiments which shows that the difference between the adiabatic flame temperature and the fiber melting point become smaller as the total flow rate increases. However, in our experiments, the total gas flow rate was increased by increasing the hydrogen flow rate which also increased the flame temperature. Hence, the conductive heat loss to the burner was determined by a competition between the reduced heat transfer coefficient and the enhanced flame temperature, which seemed to favor the reduction of the heat transfer rate by increased flow rate. Indeed, it is remarkable that the hydrogen flow rate at which ytrria's melting was observed $(-2700 \mathrm{~K})$ gives an adiabatic flame temperature which is so close to ytrria's melting point. This observation also provides strong evidence that radiative heat loss from the fiber is small.

Despite the remarkable reproducibility and improvements in the uncertainty levels of this study at such high temperatures, a key question is still the accuracy of our technique in determining the actual gas temperatures. Addressing this question requires knowledge of how much cooler the fiber can be than its surrounding gas. Hence, we took two separate approaches to better quantify the effect of radiative cooling experienced by the fibers. The first one was a theoretical approach based on the available optical data for alumina. The fiber emittance, $e$, is proportional to the fiber diameter, $d$, by the relationship $e \sim 1-\exp (-k d)$, where $k$ is the absorption coefficient of the material. This relationship shows that as the thickness of the material gets smaller its emittance becomes smaller, or equivalently, its transmittance gets larger. When the material becomes optically thick enough to be opaque, i.e. $\mathrm{kd}>>1$, then the material is no longer transparent, and the emittance is then known as the emissivity. The absorption coefficient of alumina is reported in the literature as a function of wavelength at various temperatures [8]. We utilized this absorption coefficient information at $2293 \mathrm{~K}$, combined with the spectral radiance function, to calculate the total emittance of a $125 \mu \mathrm{m}$-diameter alumina fiber close to its melting point [14]. The calculated total emittance value was 0.008 . Naturally, this value gets even smaller for thinner alumina fibers. Even if the actual emittance is larger than our calculated value by a factor of 5 , we estimate a radiative correction, using standard heat transfer correlations [15], of less than $15 \mathrm{~K}$. Since the other fibers are also oxide materials with similar visible appearance and behavior as that of alumina, and ceramics are relatively poor conductors of heat, we believe that this correction is realistic for the conditions of our experiment. 
Our second approach to characterize the significance of radiative cooling of the fibers was an experimental one. We varied the diameters of our original fibers from $60 \mu \mathrm{m}$ to $160 \mu \mathrm{m}$. During the fiber-tip melting, re-solidification, and re-melting cycles of our experiments, we observed that the fiber's tip diameter became even larger than $400 \mu \mathrm{m}$. The standard deviation of our experimental results for all fiber sizes, however, was within the uncertainty in the fiber melting temperatures. This was despite the fact that emittance is exponentially dependent on the size of the sample, as given by the relationship above, and the fiber tip size covered a range of almost one order of magnitude. For example, for the more-readily-available alumina fiber, we used at least five fibers from each of the three original-diameter classes of $60 \mu \mathrm{m}-80 \mu \mathrm{m}, 90 \mu \mathrm{m}-120 \mu \mathrm{m}$, and $130 \mu \mathrm{m}-160 \mu \mathrm{m}$. Such independence also confirms that the effects of temperature gradients across the fiber diameter and the amount of latent heat required to melt the fiber tip are negligible. We interpret this finding to mean that the emittance of the fibers was so small in our experiments that any variation of emittance with respect to the size of the fiber tip was encompassed by our experimental reproducibility with an uncertainty of $\pm 15 \mathrm{~K}$. Hence, we conclude that the determination of actual gas temperatures requires a correction of not more than $15 \mathrm{~K}$ to be added to the directly measured high temperatures obtained using our technique.

We applied our technique first to assess the accuracy of our thermocouple measurements. The results are depicted in Fig. 5. We used only three lower-melting-point materials for this purpose since the temperatures required for yttria are too high for our thermocouple to survive. The curve labeled "material's melting point" represents the accurate reference temperatures to which our thermocouple measurements need to be compared. The curve labeled "T/C corrected" represents the gas temperatures inferred from thermocouple measurements after applying corrections for both radiative and conductive cooling of the bead. The thermal and radiative properties of thermocouple materials, rhodium and iridium, are obtained from [16], and an emissivity value of 0.35 was judged to be a reasonable estimate for the conditions of our experiment. The measured gas- temperature gradient in the radial direction is used when applying the conduction correction. Figure 5 confirms two major points. The first point is that substantial corrections are required to thermocouple measurements which are typically larger than $150 \mathrm{~K}$ at such high temperatures. Secondly, these corrections are accompanied by large uncertainties due to uncertainties in material properties used and heat transfer correlations applied, such that one can still be off from the actual gas temperature by more than $100 \mathrm{~K}$ even after the corrections. The consistent underprediction of thermocouple measurements for all three materials suggests a systematic error, which can be due to, say, the emissivity value used. Yet, without our reference measurements, such suspicions would only be a part of the uncertainties associated with corrections.

We then applied our technique to calibrate the IR camera discussed above. The calibration curve obtained is depicted in Fig. 6. The error bars indicate the uncertainty in our technique in determining the gas temperature on the horizontal axis, and the overall uncertainty of $\pm 15 \%$ in the band-ratio measurements on the vertical axis. Unfortunately, at the time of our experiments, we did not have proper neutral density filters to be able to take $\mathrm{H}_{2} \mathrm{O}$ emission data to determine the band ratio at the high flame temperatures relevant to yttria. Therefore, we extrapolated the calibration curve by a dashed line only for the purpose of demonstrating a potential application of 
our technique. Emission data taken by using either pure oxygen or air for generating $\mathrm{H}_{2} \mathrm{O}$ shows the consistency of also the band ratio measurements, just as was the case for the fiber melting point measurements. Given the uncertainties in the measured band ratios and the slope of the calibration curve, the accuracy of the IR camera we calibrated was about $\pm 65 \mathrm{~K}$.

\section{CONCLUDING REMARKS}

A technique is described to measure gas temperatures above $2000 \mathrm{~K}$ with an accuracy and reproducibility estimated to be within $\pm 15 \mathrm{~K}$. Pure, transparent, thin metal-oxide materials were utilized which had abrupt changes in their emittance upon melting. These materials were in fiber forms and had known melting points up to $2690 \mathrm{~K}$. Hydrogen-air and/or hydrogen-oxygen flames were used to obtain the high temperatures. The gas temperatures could be adjusted and controlled within $4 \mathrm{~K}$. The experimental melting-point measurements were independent of fiber diameters, which varied from $60 \mu \mathrm{m}$ to $400 \mu \mathrm{m}$ in our experiments. The calculated emittance values, based on published absorption coefficient data, were also of order 0.01 at temperatures a few degrees below the melting point. These findings indicated that radiative cooling of fibers was negligible prior to melting, allowing direct measurement of gas temperature. The accuracy of the method was limited by how well the melting points of these materials were known. The technique was applied first to assess the accuracy of gas-temperature measurements inferred from thermocouples, and confirmed that such measurements are typically accompanied by uncertainties larger than $100 \mathrm{~K}$. The technique was also used to calibrate an IR camera to measure gas temperatures using the band-ratio method for hot water emission at $1.45 \mu \mathrm{m}$ and $1.94 \mu \mathrm{m}$. It was demonstrated that the technique offers an excellent calibration reference for other gas-temperature measurement methods to improve their accuracy at higher temperatures.

\section{REFERENCES}

[1] Xing, Y., Koylu, U.O., and Rosner, D.E., "Synthesis and Restructuring of Inorganic NanoParticles in Counterflow Diffusion Flames," Combust. \& Flame, 107, 85 (1996).

[2] Prucker, S., Meier, W., and Stricker, W., "A Flat Flame Burner as Calibration Source for Combustion Research: Temperature and Species Concentrations of Premixed $\mathrm{H}_{2} / \mathrm{Air}$ Flames," Rev. Sci. Instrum. 65 (9), 2908-2911 (1994).

[3] Weast, R.C. and Astle, M.J., Handbook of Physics and Chemistry (59 $\left.9^{\text {th }} \mathrm{ed}\right)$, CRC Press, Boca Raton, 1979, p B-91.

[4] Levin, E.M. and McMurdie, H.F., Phase Diagrams for Ceramists - 1975 Supplement, Reser, M.K. (ed), American Ceramic Society, Columbus, 1975, p 132.

[5] Bedford, R.E., Bonnier, G., Maas, H., and Pavese, F., "Recommended Values of Temperature on the International Temperature Scale of 1990 for a Selected Set of Secondary Reference Points," Metrologia, 33, 133-154 (1996).

[6] Sayir, A. and Farmer, S.C., "Directionally Solidified Mullite Fibers," Ceramic Matrix Composites - Advanced High-Temperature Structural Materials, Lowden, R.A., et al. (eds), Mat. Res. Soc. Proc., 365 (1995), 11-21.

[7] Nason, D.O., Yen, C.T., and Tiller, W.A., "Measurement of Optical Properties of Some Molten Oxides," J. of Crystal Growth, 106, 221-226 (1990). 
[8] Gryvnak, D.A. and Burch, D.E., "Optical and Infrared Properties of $\mathrm{Al}_{2} \mathrm{O}_{3}$ at Elevated Temperatures," J. of the Optical Society of America, 55 (6), 625-629 (1965).

[9] Lewis, B. and Von Elbe, G., Combustion, Flames and Explosions of Gases ( $\left.3^{\text {rd }} \mathrm{ed}\right)$, Academic Press, Boston, 1987, p 396.

[10] Glassman, I., Combustion (2 ${ }^{\text {nd }}$ ed), Academic Press, Boston, 1987, p 138.

[11] Gordon, S. and McBride, B.J., "Computer Program for Calculation of Complex Chemical Equilibrium Compositions and Applications," NASA Reference Publication 1311, NASA Lewis Research Center, 1994.

[12] Ferriso, C.C., Ludwig, C.B., and Boynton, F.P., “A Band-Ratio Technique for Determining Temperatures and Concentrations of Hot Combustion Gases from Infrared-Emission Spectra," Tenth Symposium (Int.) on Combustion, The Combustion Institute, Pittsburgh, PA, 1965, pp 161-175.

[13] Svehla, R.A., "Estimated Viscosities and Thermal Conductivities of Gases at High Temperatures" NASA Technical Report 132 (1962).

[14] Bass, M., Handbook of Optics ( $2^{\text {nd }}$ ed), Vol. 1, McGraw Hill, New York, 1995, Ch. 44.

[15] Kays, W.M. and Crawford, M.E., Convective Heat and Mass Transfer ( $2^{\text {nd }}$ ed), McGraw Hill, New York, 1980, p 157.

[16] Touloukian, Y.S. and DeWitt, D.P., Thermophysical Properties of Matter, Vols. 1 \& 7 , IFI/Plenum, New York, 1970.

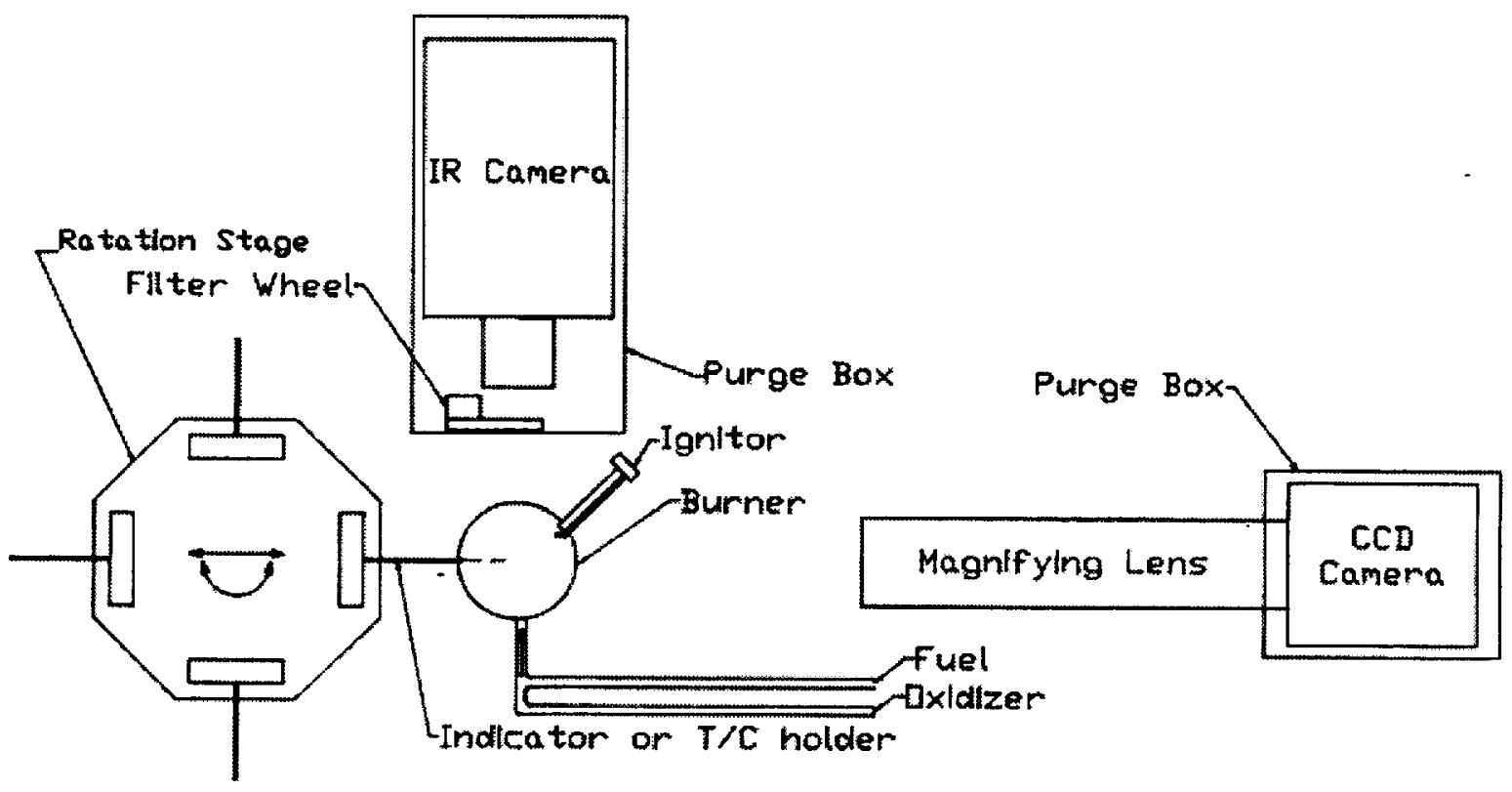

Figure 1. The schematic of experimental setup. 


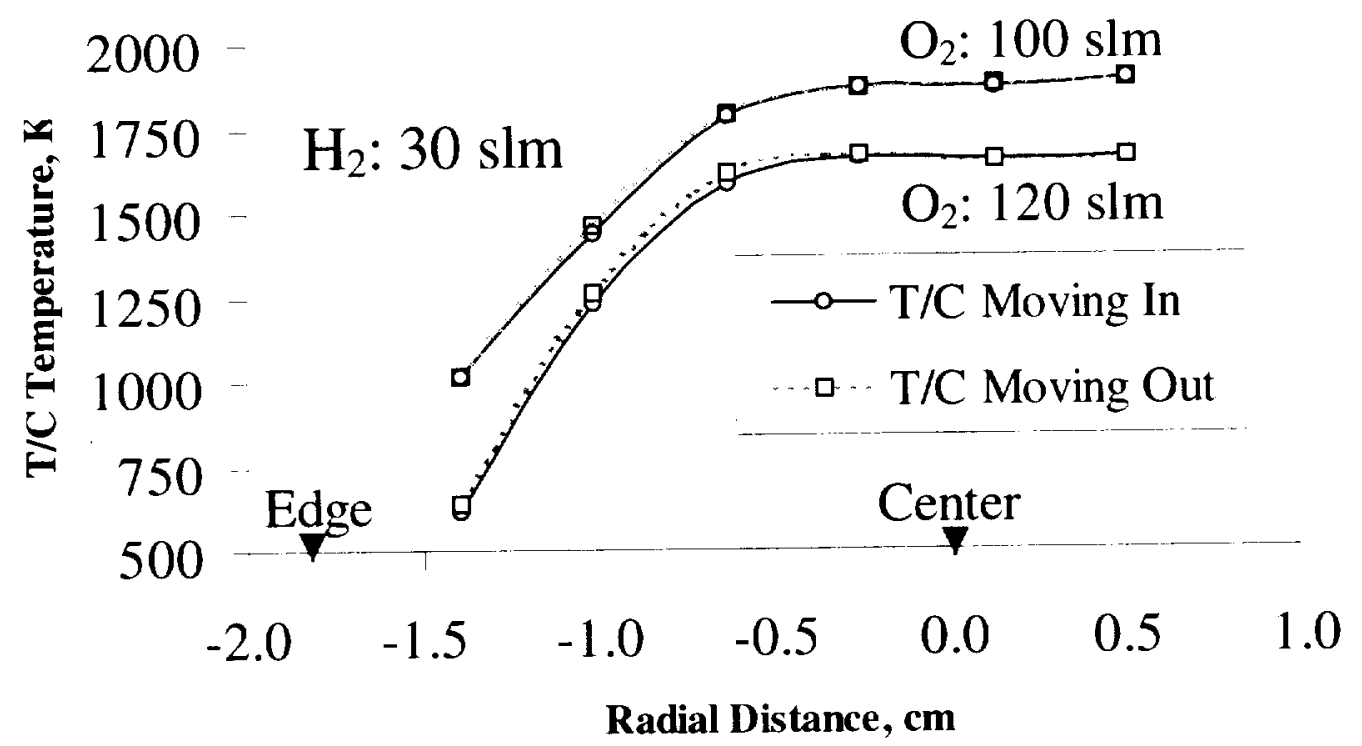

Figure 2. Temperature profiles measured by thermocouples traversing a radius of flame $3 \mathrm{~cm}$ above the burner.

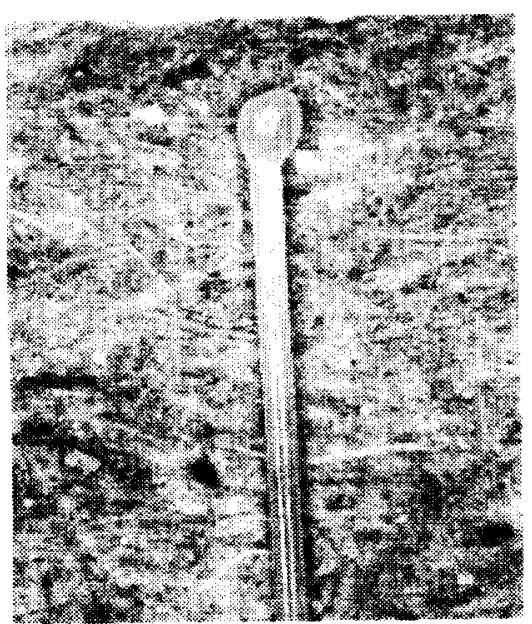

Alumina/YAG eutectic

(Melting point: 2095K)

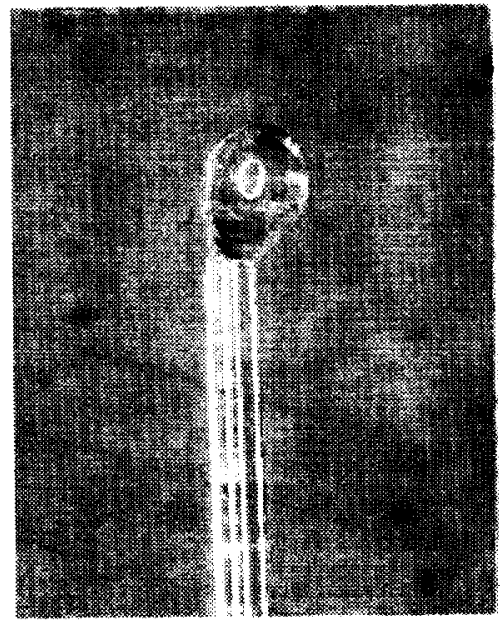

YAG

(Melting point: $2200 \mathrm{~K}$ )

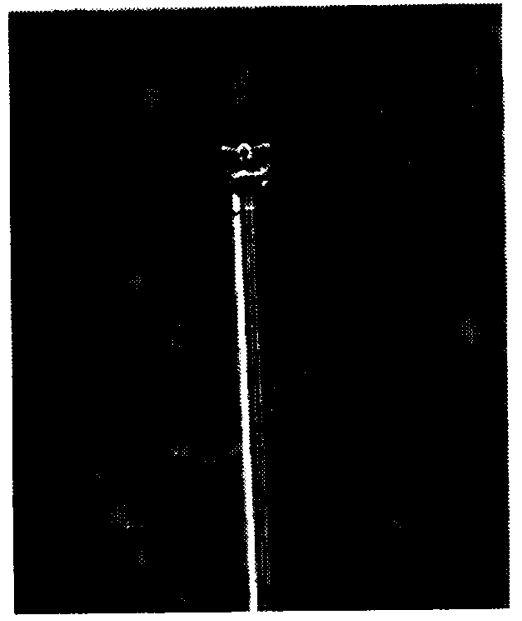

Alumina

(Melting point: $2320 \mathrm{~K}$ )

Figure 3. Microscope photographs (30X magnified) of molten fiber tips after re-solidification. 


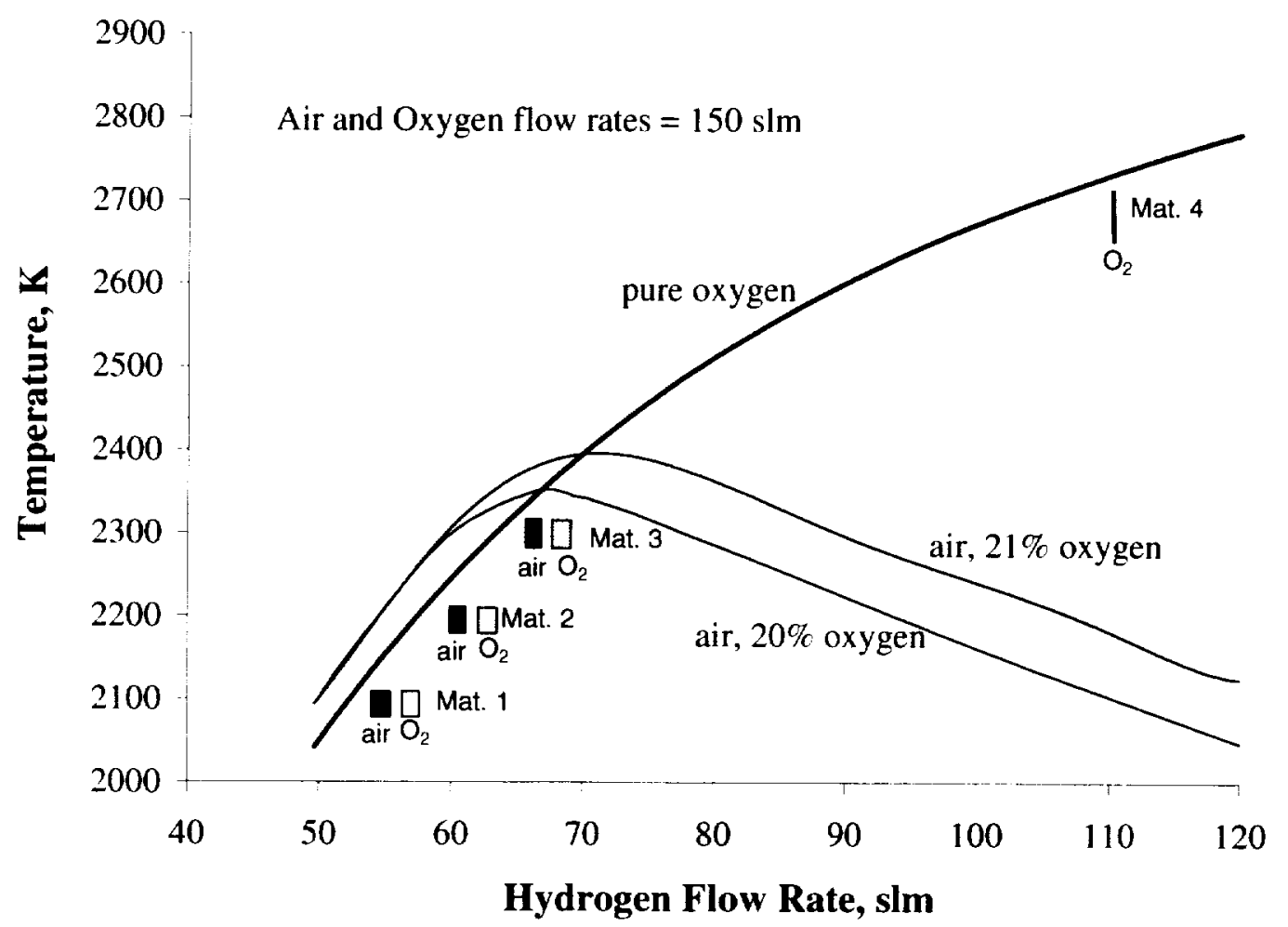

Figure 4. Comparison of adiabatic flame temperatures to melting-point measurements. Material type 1: Alumina/YAG eutectic; 2: YAG; 3: Alumina; 4: Yttria.

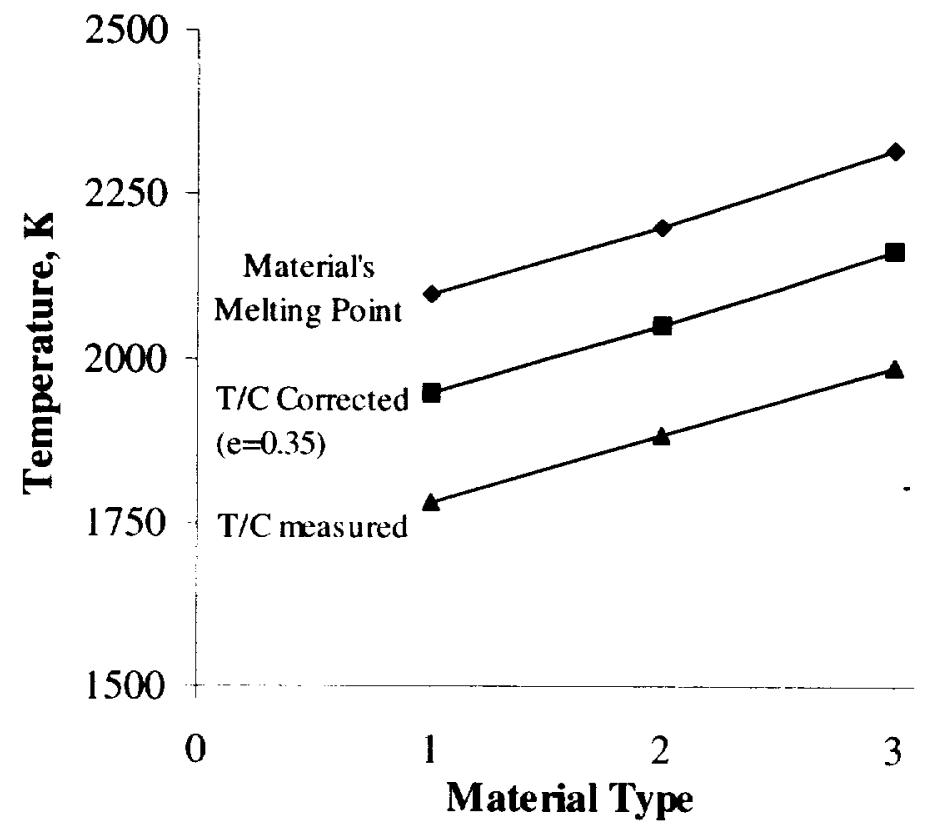

Figure 5. Temperatures measured and inferred by thermocouples versus "reference" melting-point measurements. Material type 1: Alumina/YAG eutectic; 2: YAG; 3: Alumina. 


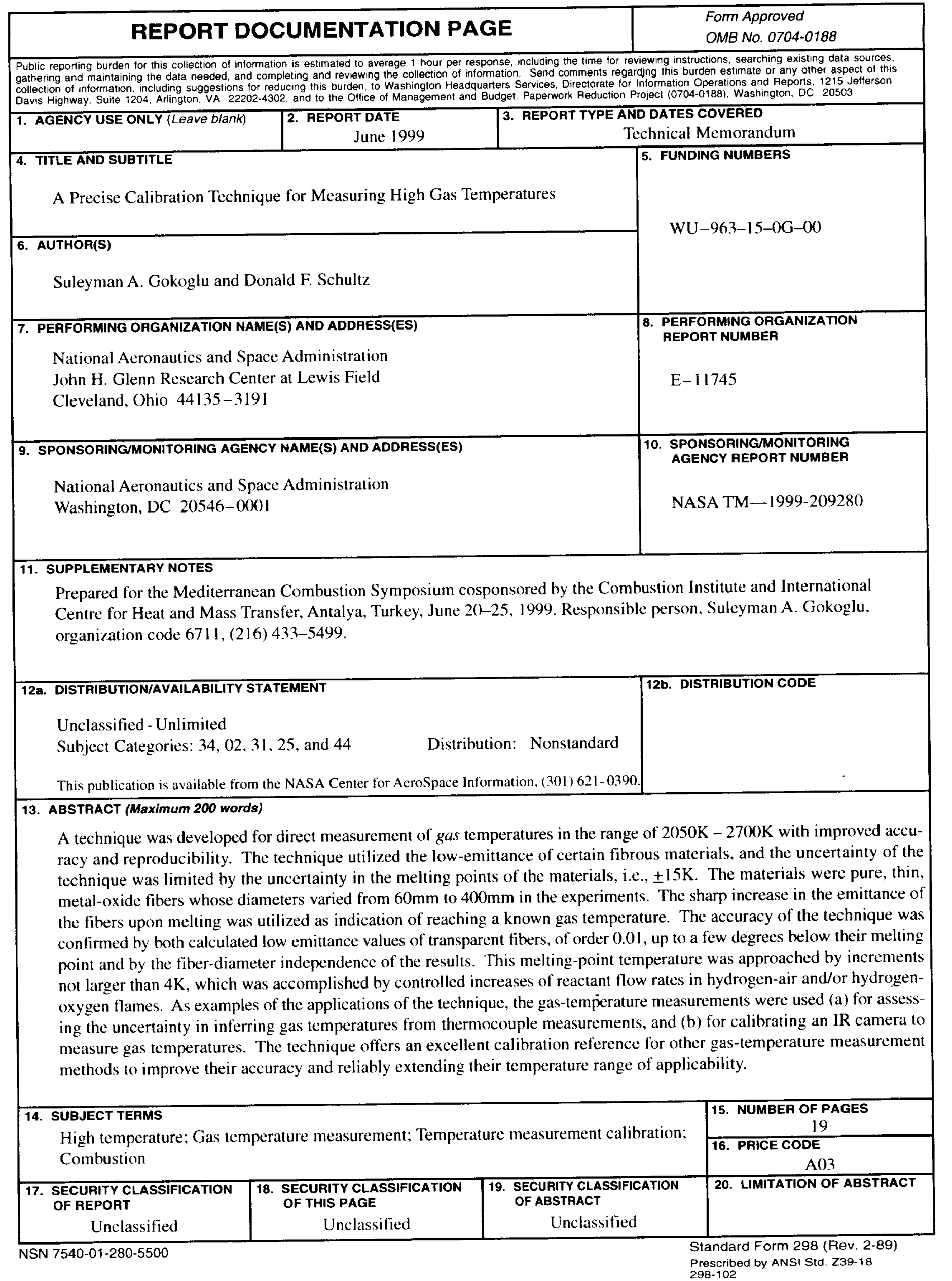




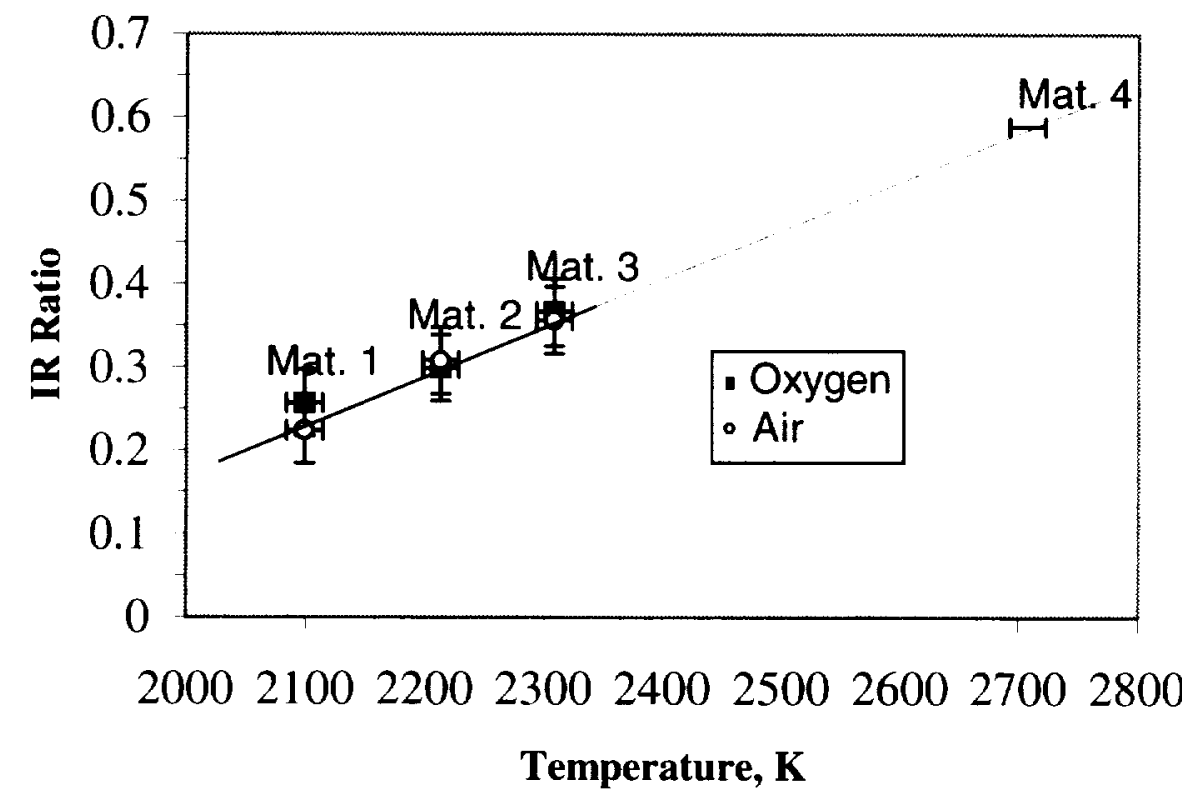

Figure 6. Calibration curve for the IR camera. Material type 1: Alumina/YAG eutectic; 2: YAG; 3: Alumina; 4: Yttria. 